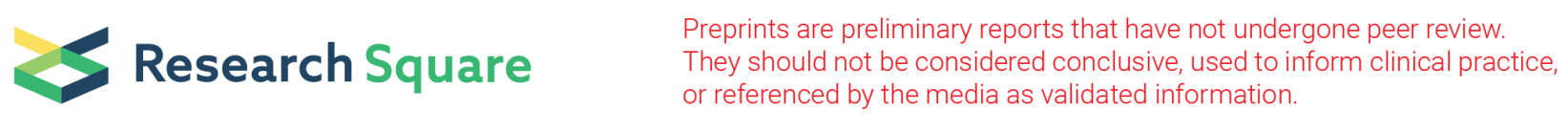

\title{
Impact of the COVID-19 Pandemic on Willingness to Adopt Healthy Dietary Habits in China
}

\section{Ying $\mathrm{Xu}$ \\ Guangdong Medical University \\ Zhixue Li \\ Guangdong Medical University \\ Weijun Yu \\ Guangdong Medical University}

Ting Liu

Guangdong Medical University

Zheng Liu

Guangdong Medical University

Liuna Yang

Guangdong Medical University

Ailun Xie

Guangdong Medical University

Zhenzhu Qian

Guangdong Medical University

Rencheng Zhao

Guangdong Medical University

YanFang Guo

Centre for Chronic Disease Control

Jialong Chen ( $\square$ chenjialongaa@163.com)

Guangdong Medical University https://orcid.org/0000-0001-5040-0692

\section{Research article}

Keywords: COVID-19, epidemic, diet, subjective impact, cross-sectional study

Posted Date: February 17th, 2021

DOl: https://doi.org/10.21203/rs.3.rs-243910/v1

License: () (1) This work is licensed under a Creative Commons Attribution 4.0 International License. Read Full License 


\section{Abstract}

Background: To investigate impact of the 2019 novel coronavirus disease (COVID-19) pandemic on willingness to adopt healthy dietary habits in China.

Methods: A survey was carried out, and subjective perception of impact due to COVID-19 and willingness to change dietary habits were obtained.

Results: A total of 22,459 subjects were derived from China, with an average age of $27.9 \pm 7.8$ years old. Of them, the mean score of willingness to adopt healthy dietary habits was 2.2 (ranges from -9 to 9). Multivariate regression analysis showed that the impact of the COVID-19 pandemic (epidemic concern, impact of psychology, impact of work or study) are associated with a higher score of willingness to adopt healthy dietary habits among female, the older, onmedical worker, and individuals married or with higher education level, normal BMI.

Conclusions: There was a positive improvement to a proper diet, so the changing features of diets should be considered in nutritional interventions for maintaining health, and prevention and control COVID-19 during the pandemic period.

\section{Background}

Since 11 March 2020, COVID-19 has spread to cause a global pandemic for more than two months and has yet to be brought under control ${ }^{1,2}$. It is generally accepted that older people, those with underlying medical conditions, are susceptible to COVID-19 ${ }^{3}$ and have a poor prognosis ${ }^{4}$. Lifestyle risk factors of noncommunicable disease including unhealthy diet, physical inactivity, smoking, obesity, heavy alcohol consumption have been consistently associated with morbidity, mortality and loss of disease-free years of life ${ }^{5}$. Meanwhile, there are also population cohort data on possible adverse effects of poor lifestyle on serious COVID-19 infections. For example, Mark Hamer et al. found a dose-dependent increase in risk of COVID-19 with less favorable lifestyle scores, such that participants in the most adverse category had 4-fold higher risk $(4.41 ; 95 \% \mathrm{Cl}: 2.52-7.71)$ compared to people with the most optimal lifestyle ${ }^{6}$. The population attributable fraction of severe COVID-19 for the three unhealthy lifestyle factors in combination was $51.4 \%$ ( $13.3 \%$ for smoking, $8.6 \%$ for physical inactivity, and $29.5 \%$ for overweight and obesity $)^{6}$. Furthermore, peripheral inflammation caused by COVID-19 may have long-term consequences in those that recover, leading to chronic medical conditions such as dementia and neurodegenerative disease, which is involved in neuroinflammatory mechanisms that can be compounded by lifestyle habits, such as consumption of unhealthy diets ${ }^{7}$.

In order to fight COVID-19, some effective personal hygiene practices were adopted by the general public. Lorene M. Nelson et al. reported that most (95.7\%) respondents were making changes to their lifestyle, mainly including more hand washing (93.1\%), avoiding social gatherings (89.0\%), and stockpiling food and supplies in response to COVID-19 $(74.7 \%)^{8}$. Kin On Kwok et al. also reported that most respondents (> 89\%) adopted enhanced personal hygiene practices including wearing masks, cleaning their hands, and adopting better coughing and sneezing etiquette and avoided traveling ${ }^{9}$. Obviously, people in general around the world have tended to change their lifestyles in response to the virus. However, whether health-related dietary habits, including eating healthier diets, has been considered effective to decrease the risk of chronic diseases ${ }^{10}$ to reduce serious outcomes of COVID-19 infections, were or will be adopted by general residents is still unknown.

According to Transtheoretical Model (TTM), there are five relatively independent but interrelated stages, including precontemplation, contemplation, preparation, action, and maintenance, of healthy-related behaviors through the process of behavior change ${ }^{11}$. Thus, our study aimed to investigate willingness to adopt healthy dietary habits in a short time of the COVID-19 pandemic. In this study, we describe characteristics of subjective perception of impact during the pandemic, and the association between subjective perception of impact and willingness to adopt healthy dietary habits.

\section{Methods}

\subsection{Participants}

The cross-sectional study was conducted online for all netizens in China in March of 2020. The participants were recruited by the snowball sampling methods: (1) they volunteered to participate in this questionnaire survey, and (2) they independently completed the questionnaire with no logical errors. A total of 28,877 respondents in China completed their questionnaires in the study. Among them, 1,966 dropped out of the study, and 26,911 validly completed their questionnaires.

\subsection{Data collection}

All questionnaires were completed online by logging onto a web address or scanning a QR code. At the beginning of the survey, we used a unified guidance language to introduce the study purposes and also ensured data confidentiality to the respondents. When there was any omission or logical error, the system prompted the respondent until the questionnaire was completed and submitted. The online questionnaires were anonymous so that the respondents could not be affected by any other factors in expressing their opinions, which helped to obtain more authentic and reliable data than traditional paper questionnaires. Of the 26,911 respondents, 4,452 invalid questionnaires (with logical errors) were excluded, and finally 22,459 were effective; the effective rate was $83.5 \%$.

\subsection{Questionnaire}

A structured questionnaire with close-ended questions was developed after an extensive literature search and consulting experts, and included the following:

(1) General characteristics: gender, age, height, weight, education level, marital status, occupation, main living place during the outbreak of CoVID-19, etc.; (2) Subjective perception of impacts: self-rated perception (none, low, medium, high, very high) of subjective impact, including epidemic concern and impact on psychology, life, work during the outbreak of COVID-19; (3) Baseline of dietary habits before the COVID-19 outbreak: salt intake (high, medium, and low), intake frequency of different food groups (times per week) which consisted of vegetables, fresh fruits, meat, dairy, and eggs, sugary foods, pickled fruits processed 
meat, drinking; (4) Willingness to change dietary habits: self-reported willingness (unchanged, increase, decrease, uncertain) to change food intake during the COVID-19 outbreak or later.

\subsection{Recode of baseline diet habits and Change willingness}

Baseline of dietary intake was recoded refer to the Chinese Diet Balance Index (DBI), including total score (DBI-TS), lower bound score (DBI-LBS), higher bound scores (DBI-HBS) (Table 1). The score and scoring method for each item of food were determined according to the recommendation by The Dietary Guidelines for Chinese Residents. A negative score is related to malnutrition; on the contrary, a positive score is related to over-nutrition. DBI-TS was obtained by the cumulative scores of all types of foods, which reflected to the average level of overall dietary quality. DBI-LBS was obtained by the cumulation of the negative scores among all types of foods, which reflected to the characteristic of insufficient intake in diet. DBI-HBS was obtained by the cumulation of the positive scores among all types of foods, which reflected to the characteristic of excessive intake in diet. Willingness to change dietary habits were classified into numerical variable (including "worse" was defined as -1 , "unsure" or "unchanged" was defined as 0, "better" were defined as 1) (Table 1). 
Table 1

Recode of diet habits during the univariate and multivariate analysis

\begin{tabular}{|c|c|c|c|c|c|}
\hline \multirow[t]{2}{*}{ Foods } & \multicolumn{2}{|l|}{ Baseline of habits } & \multicolumn{3}{|l|}{ Change willingness } \\
\hline & Intake or intake frequency & Code & Categories & Code & Implications \\
\hline \multicolumn{6}{|c|}{ Vegetables } \\
\hline & Every day & 0 & Unchange or unsure & 0 & Unchange \\
\hline & 4-6 days/week & -2 & Increase & 1 & Better \\
\hline & 2-3 days/week & -4 & Decrease & -1 & Worse \\
\hline & $<1$ days/week & -6 & & & \\
\hline \multicolumn{6}{|c|}{ Fresh Fruits } \\
\hline & Every day & 0 & Unchange or unsure & 0 & Unchange \\
\hline & 4-6 days/week & -2 & Increase & 1 & Better \\
\hline & 2-3 days/week & -4 & Decrease & -1 & Worse \\
\hline & $<1$ days/week & -6 & & & \\
\hline \multicolumn{6}{|c|}{ Meat, Dairy, And Eggs } \\
\hline & Every day & 0 & Unchange or unsure & 0 & Unchange \\
\hline & 4-6 days/week & -2 & Increase & 1 & Better \\
\hline & 2-3 days/week & -4 & Decrease & -1 & Worse \\
\hline & $<1$ days/week & -6 & & & \\
\hline \multicolumn{6}{|c|}{ Salt Intake } \\
\hline & High & 6 & Unchange or unsure & 0 & Unchange \\
\hline & Medium & 3 & Increase & -1 & Worse \\
\hline & Low & 0 & Decrease & 1 & Better \\
\hline \multicolumn{6}{|c|}{ Fried Foods } \\
\hline & Every day & 6 & Unchange or unsure & 0 & Unchange \\
\hline & 4-6 days/week & 4 & Increase & -1 & Worse \\
\hline & 2-3 days/week & 2 & Decrease & 1 & Better \\
\hline & $<1$ days/week & 0 & & & \\
\hline \multicolumn{6}{|c|}{ Sugary Foods } \\
\hline & Every day & 6 & Unchange or unsure & 0 & Unchange \\
\hline & 4-6 days/week & 4 & Increase & -1 & Worse \\
\hline & 2-3 days/week & 2 & Decrease & 1 & Better \\
\hline & $<1$ days/week & 0 & & & \\
\hline \multicolumn{6}{|c|}{ Pickled Fruits } \\
\hline & Every day & 6 & Unchange or unsure & 0 & Unchange \\
\hline & 4-6 days/week & 4 & Increase & -1 & Worse \\
\hline & 2-3 days/week & 2 & Decrease & 1 & Better \\
\hline & $<1$ days/week & 0 & & & \\
\hline \multicolumn{6}{|c|}{ Processed Meat } \\
\hline & Every day & 6 & Unchange or unsure & 0 & Unchange \\
\hline & 4-6 days/week & 4 & Increase & -1 & Worse \\
\hline & 2-3 days/week & 2 & Decrease & 1 & Better \\
\hline & $<1$ days/week & 0 & & & \\
\hline
\end{tabular}




\begin{tabular}{|llllll|}
\hline Drinking & & & & & \\
& Every day & 6 & Unchange or unsure & 0 & Unchange \\
\hline $4-6$ days/week & 4 & Increase & -1 & Worse \\
\hline $2-3$ days/week & 2 & Decrease & 1 & Better \\
\hline$<1$ days/week & 0 & & & \\
\hline Code: code in univariate and multivariate analysis & & & & \\
\hline
\end{tabular}

\subsection{Statistical methods}

The original data of the questionnaires filled in online were downloaded directly from the website, and all the data were imported into SPSS18.0 software for statistical analysis after the invalid questionnaires were removed. Measurement data (age and BMI) followed a normal distribution, were described using the arithmetical means and standard deviation, and were analyzed by the Student's t-test or $\mathrm{F}$ test to compare the means between different groups. Categorical data were described as the proportion and analyzed with the chi-square test. Stepwise linear regression models $(p$ in $<0.05, p$ out $>0.10)$ were fitted to explore the factors influencing total score of willingness to change dietary habits. In multivariate stepwise linear regression analysis, gender, marital status, occupation, main living place was recoded to dichotomous variables, and BMI, education level was treated as ordered categorical variables, and subjective perception of impacts were classified into dichotomous variables ("none," "low," and "medium" were defined as 1, while "high" and "very high" were defined as 2) (Table 1). For all analyses, a p-value $<0.05$ was regarded as statistically significant.

\section{Results}

\subsection{General information}

A total of 22,459 subjects were included in this study, including 14,204 males (63.2\%) and 8,255 females (36.8\%). Among them, 11,182 (49.8\%) were married, and 10,567 (47.1\%) were unmarried; 20,650 (91.9\%) were in senior high school and above and 1,809 (8.1\%) in junior high school and below; only 711 (3.2\%) were medical workers. The mean age and BMI were $27.9 \pm 7.8$ (years) and $22.1 \pm 4.9$, respectively. During the outbreak of COVID-19, 14,069 (62.6\%) lived in Guangdong Province, 292 (1.3\%) lived in Hubei, the area with severe epidemics in China, and 8,098 (36.1\%) in other provinces (Table 1).

\subsection{Subjective perception of impact}

Nearly one-third and two-fifths of self-reported status of epidemic concern were relatively high (32.5\%) and very high (42.5\%), respectively. Over $60.0 \%$ selfreported a subjective impact (middle or above) on psychology $(60.2 \%)$, life (66.3\%), and work (66.8\%). In different genders, the difference in subjective impacts was statistically significant $(\mathrm{P}<0.05)($ Table 2$)$. 
Table 2

Willingness to change dietary habits during the pandemic of COVID-19

\begin{tabular}{|c|c|c|c|c|c|c|c|c|c|c|c|c|}
\hline Characteristic & $N(\%)$ & Vegetables & $\begin{array}{l}\text { Fresh } \\
\text { Fruits }\end{array}$ & $\begin{array}{l}\text { Meat, } \\
\text { Dairy, } \\
\text { And } \\
\text { Eggs }\end{array}$ & $\begin{array}{l}\text { Salt } \\
\text { Intake }\end{array}$ & $\begin{array}{l}\text { Fried } \\
\text { Foods }\end{array}$ & $\begin{array}{l}\text { Sugary } \\
\text { Foods }\end{array}$ & $\begin{array}{l}\text { Pickled } \\
\text { Fruits }\end{array}$ & $\begin{array}{l}\text { Processed } \\
\text { Meat }\end{array}$ & Drinking & $\begin{array}{l}\text { Total } \\
\text { score }\end{array}$ & Cc \\
\hline \multicolumn{13}{|l|}{$\begin{array}{l}\text { General } \\
\text { characteristics }\end{array}$} \\
\hline \multicolumn{13}{|l|}{ Gender } \\
\hline Male & $14204(63.2 \%)$ & $0.32 \pm 0.70$ & $\begin{array}{l}0.30 \pm \\
0.72\end{array}$ & $\begin{array}{l}0.17 \pm \\
0.73\end{array}$ & $\begin{array}{l}0.11 \pm \\
0.68\end{array}$ & $\begin{array}{l}0.20 \pm \\
0.77\end{array}$ & $\begin{array}{l}0.14 \pm \\
0.77\end{array}$ & $\begin{array}{l}0.16 \pm \\
0.77\end{array}$ & $\begin{array}{l}0.13 \pm \\
0.77\end{array}$ & $\begin{array}{l}0.25 \pm \\
0.68\end{array}$ & $\begin{array}{l}1.79 \pm \\
3.16\end{array}$ & 1 \\
\hline Female & $8255(36.8 \%)$ & $0.39 \pm 0.68$ & $\begin{array}{l}0.36 \pm \\
0.69\end{array}$ & $\begin{array}{l}0.25 \pm \\
0.71\end{array}$ & $\begin{array}{l}0.25 \pm \\
0.65\end{array}$ & $\begin{array}{l}0.37 \pm \\
0.74\end{array}$ & $\begin{array}{l}0.31 \pm \\
0.74\end{array}$ & $\begin{array}{l}0.35 \pm \\
0.74\end{array}$ & $\begin{array}{l}0.31 \pm \\
0.74\end{array}$ & $\begin{array}{l}0.24 \pm \\
0.6\end{array}$ & $\begin{array}{l}2.84 \pm \\
3.39\end{array}$ & 2 \\
\hline$T$ & & -6.456 & -6.095 & -7.543 & -15.702 & -16.199 & -16.777 & -18.004 & -17.098 & 0.715 & -23.365 & \\
\hline$P$ value & & $<0.001$ & $<.001$ & $<0.001$ & $<0.001$ & $<0.001$ & $<0.001$ & $<0.001$ & $<0.001$ & 0.475 & $<0.001$ & \\
\hline \multicolumn{13}{|l|}{ Age(Year) } \\
\hline$\leq 20$ & $4282(19.1 \%)$ & $0.28 \pm 0.64$ & $\begin{array}{l}0.25 \pm \\
0.66\end{array}$ & $\begin{array}{l}0.12 \pm \\
0.67\end{array}$ & $\begin{array}{l}0.05 \pm \\
0.58\end{array}$ & $\begin{array}{l}0.18 \pm \\
0.71\end{array}$ & $\begin{array}{l}0.10 \pm \\
0.70\end{array}$ & $\begin{array}{l}0.12 \pm \\
0.69\end{array}$ & $0.08 \pm 0.7$ & $\begin{array}{l}0.17 \pm \\
0.59\end{array}$ & $\begin{array}{l}1.34 \pm \\
2.64\end{array}$ & 1 \\
\hline $20-$ & $5782(25.7 \%)$ & $0.31 \pm 0.72$ & $\begin{array}{l}0.30 \pm \\
0.73\end{array}$ & $\begin{array}{l}0.17 \pm \\
0.74\end{array}$ & $\begin{array}{l}0.08 \pm \\
0.68\end{array}$ & $\begin{array}{l}0.19 \pm \\
0.79\end{array}$ & $\begin{array}{l}0.12 \pm \\
0.79\end{array}$ & $\begin{array}{l}0.13 \pm \\
0.78\end{array}$ & $\begin{array}{l}0.10 \pm \\
0.78\end{array}$ & $\begin{array}{l}0.20 \pm \\
0.68\end{array}$ & $\begin{array}{l}1.61 \pm \\
3.01\end{array}$ & 2 \\
\hline $25-$ & $5351(23.8 \%)$ & $0.39 \pm 0.70$ & $\begin{array}{l}0.36 \pm \\
0.72\end{array}$ & $\begin{array}{l}0.25 \pm \\
0.73\end{array}$ & $\begin{array}{l}0.21 \pm \\
0.69\end{array}$ & $\begin{array}{l}0.30 \pm \\
0.78\end{array}$ & $\begin{array}{l}0.24 \pm \\
0.77\end{array}$ & $\begin{array}{l}0.27 \pm \\
0.78\end{array}$ & $\begin{array}{l}0.23 \pm \\
0.78\end{array}$ & $\begin{array}{l}0.28 \pm \\
0.66\end{array}$ & $\begin{array}{l}2.51 \pm \\
3.44\end{array}$ & 3 \\
\hline$\geq 30$ & $7044(31.4 \%)$ & $0.39 \pm 0.69$ & $\begin{array}{l}0.37 \pm \\
0.7\end{array}$ & $\begin{array}{l}0.24 \pm \\
0.72\end{array}$ & $\begin{array}{l}0.26 \pm \\
0.69\end{array}$ & $\begin{array}{l}0.35 \pm \\
0.75\end{array}$ & $\begin{array}{l}0.30 \pm \\
0.75\end{array}$ & $\begin{array}{l}0.36 \pm \\
0.75\end{array}$ & $\begin{array}{l}0.31 \pm \\
0.75\end{array}$ & $\begin{array}{l}0.31 \pm \\
0.66\end{array}$ & $\begin{array}{l}2.88 \pm \\
3.54\end{array}$ & 4 \\
\hline$F$ & & 35.652 & 31.327 & 37.791 & 129.67 & 66.867 & 88.811 & 131.852 & 128.012 & 51.513 & 287.739 & \\
\hline$P$ value & & $<0.001$ & $\dot{0} .001$ & $<.001$ & $<0.001$ & $<0.001$ & $<0.001$ & $<0.001$ & $<0.001$ & $<0.001$ & $<0.001$ & \\
\hline \multicolumn{13}{|l|}{ BMI } \\
\hline$<18.5$ & $4162(18.5 \%)$ & $0.31 \pm 0.71$ & $\begin{array}{l}0.29 \pm \\
0.72\end{array}$ & $\begin{array}{l}0.19 \pm \\
0.73\end{array}$ & $\begin{array}{l}0.12 \pm \\
0.67\end{array}$ & $\begin{array}{l}0.21 \pm \\
0.78\end{array}$ & $\begin{array}{l}0.14 \pm \\
0.77\end{array}$ & $\begin{array}{l}0.16 \pm \\
0.77\end{array}$ & $\begin{array}{l}0.13 \pm \\
0.78\end{array}$ & $\begin{array}{l}0.20 \pm \\
0.67\end{array}$ & $\begin{array}{l}1.73 \pm \\
3.17\end{array}$ & 1 \\
\hline 18.5- & $12897(57.4 \%)$ & $0.37 \pm 0.69$ & $\begin{array}{l}0.34 \pm \\
0.70\end{array}$ & $\begin{array}{l}0.21 \pm \\
0.72\end{array}$ & $\begin{array}{l}0.18 \pm \\
0.67\end{array}$ & $\begin{array}{l}0.28 \pm \\
0.76\end{array}$ & $\begin{array}{l}0.22 \pm \\
0.76\end{array}$ & $\begin{array}{l}0.26 \pm \\
0.76\end{array}$ & $\begin{array}{l}0.22 \pm \\
0.76\end{array}$ & $\begin{array}{l}0.26 \pm \\
0.65\end{array}$ & $\begin{array}{l}2.34 \pm \\
3.32\end{array}$ & 2 \\
\hline$\geq 24$ & $5400(24 \%)$ & $0.33 \pm 0.69$ & $\begin{array}{l}0.32 \pm \\
0.70\end{array}$ & $\begin{array}{l}0.19 \pm \\
0.71\end{array}$ & $\begin{array}{l}0.15 \pm \\
0.66\end{array}$ & $\begin{array}{l}0.27 \pm \\
0.76\end{array}$ & $\begin{array}{l}0.20 \pm \\
0.75\end{array}$ & $\begin{array}{l}0.22 \pm \\
0.76\end{array}$ & $\begin{array}{l}0.18 \pm \\
0.76\end{array}$ & $\begin{array}{l}0.25 \pm \\
0.66\end{array}$ & $\begin{array}{l}2.12 \pm \\
3.24\end{array}$ & 3 \\
\hline$F$ & & 13.539 & 9.623 & 1.554 & 15.251 & 14.915 & 15.715 & 30.755 & 22.404 & 15.564 & 54.743 & \\
\hline$P$ value & & $<0.001$ & $<.001$ & 0.212 & $<0.001$ & $<0.001$ & $<0.001$ & $<0.001$ & $<0.001$ & $<0.001$ & $<0.001$ & \\
\hline \multicolumn{13}{|l|}{$\begin{array}{l}\text { Mainly Living } \\
\text { Place }\end{array}$} \\
\hline $\begin{array}{l}\text { Guangdong } \\
\text { Province }\end{array}$ & $14069(62.6 \%)$ & $0.35 \pm 0.71$ & $\begin{array}{l}0.32 \pm \\
0.72\end{array}$ & $\begin{array}{l}0.21 \pm \\
0.74\end{array}$ & $\begin{array}{l}0.16 \pm \\
0.69\end{array}$ & $\begin{array}{l}0.26 \pm \\
0.78\end{array}$ & $\begin{array}{l}0.20 \pm \\
0.78\end{array}$ & $\begin{array}{l}0.23 \pm \\
0.78\end{array}$ & $\begin{array}{l}0.19 \pm \\
0.78\end{array}$ & $\begin{array}{l}0.26 \pm \\
0.68\end{array}$ & $\begin{array}{l}2.18 \pm \\
3.34\end{array}$ & 1 \\
\hline Others & $8390(37.4 \%)$ & $0.34 \pm 0.67$ & $\begin{array}{l}0.33 \pm \\
0.68\end{array}$ & $\begin{array}{l}0.19 \pm \\
0.69\end{array}$ & $\begin{array}{l}0.16 \pm \\
0.64\end{array}$ & $\begin{array}{l}0.28 \pm \\
0.73\end{array}$ & $\begin{array}{l}0.21 \pm \\
0.73\end{array}$ & $\begin{array}{l}0.23 \pm \\
0.73\end{array}$ & $\begin{array}{l}0.19 \pm \\
0.73\end{array}$ & $\begin{array}{l}0.23 \pm \\
0.62\end{array}$ & $\begin{array}{l}2.15 \pm \\
3.18\end{array}$ & 2 \\
\hline$F$ & & 0.980 & -0.509 & 2.119 & 0.549 & -2.109 & -1.068 & -0.262 & 0.031 & 3.755 & 0.632 & \\
\hline$P$ value & & 0.327 & 0.61 & 0.034 & 0.583 & 0.035 & 0.286 & 0.793 & 0.975 & $<0.001$ & 0.528 & \\
\hline \multicolumn{13}{|l|}{$\begin{array}{l}\text { Education } \\
\text { Level }\end{array}$} \\
\hline $\begin{array}{l}\text { Primary } \\
\text { Schools And } \\
\text { Below }\end{array}$ & $336(1.5 \%)$ & $0.18 \pm 0.73$ & $\begin{array}{l}0.15 \pm \\
0.72\end{array}$ & $\begin{array}{l}0.15 \pm \\
0.72\end{array}$ & $\begin{array}{l}-0.09 \pm \\
0.70\end{array}$ & $\begin{array}{l}-0.02 \pm \\
0.77\end{array}$ & $\begin{array}{l}-0.08 \pm \\
0.77\end{array}$ & $\begin{array}{l}0.02 \pm \\
0.77\end{array}$ & $\begin{array}{l}0.04 \pm \\
0.76\end{array}$ & $\begin{array}{l}0.16 \pm \\
0.72\end{array}$ & $\begin{array}{l}0.51 \pm \\
2.70\end{array}$ & 1 \\
\hline $\begin{array}{l}\text { Junior High } \\
\text { School }\end{array}$ & $1473(6.6 \%)$ & $0.27 \pm 0.72$ & $\begin{array}{l}0.23 \pm \\
0.74\end{array}$ & $\begin{array}{l}0.12 \pm \\
0.75\end{array}$ & $\begin{array}{l}0.04 \pm \\
0.71\end{array}$ & $\begin{array}{l}0.15 \pm \\
0.79\end{array}$ & $\begin{array}{l}0.11 \pm \\
0.78\end{array}$ & $\begin{array}{l}0.14 \pm \\
0.79\end{array}$ & $\begin{array}{l}0.13 \pm \\
0.78\end{array}$ & $\begin{array}{l}0.20 \pm \\
0.72\end{array}$ & $\begin{array}{l}1.39 \pm \\
3.09\end{array}$ & 2 \\
\hline $\begin{array}{l}\text { High School } \\
\text { Or Technical } \\
\text { Secondary } \\
\text { School }\end{array}$ & $5874(26.2 \%)$ & $0.31 \pm 0.70$ & $\begin{array}{l}0.29 \pm \\
0.71\end{array}$ & $\begin{array}{l}0.15 \pm \\
0.72\end{array}$ & $\begin{array}{l}0.11 \pm \\
0.68\end{array}$ & $\begin{array}{l}0.22 \pm \\
0.77\end{array}$ & $\begin{array}{l}0.16 \pm \\
0.76\end{array}$ & $\begin{array}{l}0.19 \pm \\
0.76\end{array}$ & $\begin{array}{l}0.16 \pm \\
0.77\end{array}$ & $\begin{array}{l}0.24 \pm \\
0.66\end{array}$ & $\begin{array}{l}1.83 \pm \\
3.11\end{array}$ & 3 \\
\hline
\end{tabular}

Code: code in stepwise linear regression models 


\begin{tabular}{|c|c|c|c|c|c|c|c|c|c|c|c|c|}
\hline Characteristic & $N(\%)$ & Vegetables & $\begin{array}{l}\text { Fresh } \\
\text { Fruits }\end{array}$ & $\begin{array}{l}\text { Meat, } \\
\text { Dairy, } \\
\text { And } \\
\text { Eggs }\end{array}$ & $\begin{array}{l}\text { Salt } \\
\text { Intake }\end{array}$ & $\begin{array}{l}\text { Fried } \\
\text { Foods }\end{array}$ & $\begin{array}{l}\text { Sugary } \\
\text { Foods }\end{array}$ & $\begin{array}{l}\text { Pickled } \\
\text { Fruits }\end{array}$ & $\begin{array}{l}\text { Processed } \\
\text { Meat }\end{array}$ & Drinking & $\begin{array}{l}\text { Total } \\
\text { score }\end{array}$ & Cc \\
\hline College & $6507(29 \%)$ & $0.35 \pm 0.70$ & $\begin{array}{l}0.33 \pm \\
0.72\end{array}$ & $\begin{array}{l}0.20 \pm \\
0.74\end{array}$ & $\begin{array}{l}0.19 \pm \\
0.69\end{array}$ & $\begin{array}{l}0.27 \pm \\
0.77\end{array}$ & $\begin{array}{l}0.23 \pm \\
0.77\end{array}$ & $\begin{array}{l}0.26 \pm \\
0.77\end{array}$ & $0.2 \pm 0.77$ & $\begin{array}{l}0.27 \pm \\
0.67\end{array}$ & $\begin{array}{l}2.30 \pm \\
3.38\end{array}$ & 4 \\
\hline $\begin{array}{l}\text { Bachelor } \\
\text { Degree Or } \\
\text { Above }\end{array}$ & $8269(36.8 \%)$ & $0.39 \pm 0.67$ & $\begin{array}{l}0.38 \pm \\
0.68\end{array}$ & $\begin{array}{l}0.25 \pm \\
0.70\end{array}$ & $\begin{array}{l}0.20 \pm \\
0.64\end{array}$ & $\begin{array}{l}0.33 \pm \\
0.74\end{array}$ & $\begin{array}{l}0.23 \pm \\
0.75\end{array}$ & $\begin{array}{l}0.27 \pm \\
0.74\end{array}$ & $\begin{array}{l}0.23 \pm \\
0.75\end{array}$ & $\begin{array}{l}0.25 \pm \\
0.62\end{array}$ & $\begin{array}{l}2.52 \pm \\
3.32\end{array}$ & 5 \\
\hline$F$ & & 22.317 & 26.476 & 23.122 & 41.534 & 39.849 & 24.537 & 23.858 & 13.386 & 5.23 & 84.837 & \\
\hline$P$ value & & $<0.001$ & $\begin{array}{l}<.001 \\
0.001\end{array}$ & $\begin{array}{l}< \\
0.001\end{array}$ & $<0.001$ & $<0.001$ & $<0.001$ & $<0.001$ & $<0.001$ & $<0.001$ & $<0.001$ & \\
\hline \multicolumn{13}{|l|}{ Marital Status } \\
\hline Married & $11182(49.8 \%)$ & $0.39 \pm 0.70$ & $\begin{array}{l}0.37 \pm \\
0.71\end{array}$ & $\begin{array}{l}0.24 \pm \\
0.74\end{array}$ & $\begin{array}{l}0.24 \pm \\
0.70\end{array}$ & $\begin{array}{l}0.32 \pm \\
0.78\end{array}$ & $\begin{array}{l}0.26 \pm \\
0.78\end{array}$ & $\begin{array}{l}0.31 \pm \\
0.78\end{array}$ & $\begin{array}{l}0.26 \pm \\
0.78\end{array}$ & $\begin{array}{l}0.30 \pm \\
0.67\end{array}$ & $\begin{array}{l}2.70 \pm \\
3.53\end{array}$ & 1 \\
\hline Unmarried & $11277(50.2 \%)$ & $0.31 \pm 0.68$ & $\begin{array}{l}0.28 \pm \\
0.70\end{array}$ & $\begin{array}{l}0.16 \pm \\
0.71\end{array}$ & $\begin{array}{l}0.09 \pm \\
0.64\end{array}$ & $\begin{array}{l}0.21 \pm \\
0.75\end{array}$ & $\begin{array}{l}0.14 \pm \\
0.74\end{array}$ & $\begin{array}{l}0.15 \pm \\
0.74\end{array}$ & $\begin{array}{l}0.12 \pm \\
0.74\end{array}$ & $\begin{array}{l}0.20 \pm \\
0.63\end{array}$ & $\begin{array}{l}1.65 \pm \\
2.94\end{array}$ & 2 \\
\hline$F$ & & 9.216 & 9.093 & 9.145 & 16.918 & 10.146 & 11.917 & 16.124 & 14.252 & 11.849 & 24.126 & \\
\hline$P$ value & & $<0.001$ & $<.001$ & $<.001$ & $<0.001$ & $<0.001$ & $<0.001$ & $<0.001$ & $<0.001$ & $<0.001$ & $<0.001$ & \\
\hline \multicolumn{13}{|l|}{ Occupation } \\
\hline $\begin{array}{l}\text { Medical } \\
\text { Workers }\end{array}$ & $711(3.2 \%)$ & $0.24 \pm 0.78$ & $\begin{array}{l}0.28 \pm \\
0.77\end{array}$ & $\begin{array}{l}0.17 \pm \\
0.77\end{array}$ & $\begin{array}{l}0.05 \pm \\
0.75\end{array}$ & $\begin{array}{l}0.29 \pm \\
0.8\end{array}$ & $\begin{array}{l}0.17 \pm \\
0.83\end{array}$ & $\begin{array}{l}0.16 \pm \\
0.82\end{array}$ & $\begin{array}{l}0.13 \pm \\
0.82\end{array}$ & $\begin{array}{l}0.26 \pm \\
0.71\end{array}$ & $\begin{array}{l}1.75 \pm \\
3.30\end{array}$ & 1 \\
\hline Others & 21748(96.8\%) & $0.35 \pm 0.69$ & $\begin{array}{l}0.33 \pm \\
0.71\end{array}$ & $\begin{array}{l}0.20 \pm \\
0.72\end{array}$ & $\begin{array}{l}0.16 \pm \\
0.67\end{array}$ & $\begin{array}{l}0.26 \pm \\
0.76\end{array}$ & $\begin{array}{l}0.20 \pm \\
0.76\end{array}$ & $\begin{array}{l}0.23 \pm \\
0.76\end{array}$ & $\begin{array}{l}0.19 \pm \\
0.76\end{array}$ & $\begin{array}{l}0.25 \pm \\
0.65\end{array}$ & $\begin{array}{l}2.19 \pm \\
3.28\end{array}$ & 2 \\
\hline$T$ & & 16.989 & 3.024 & 1.687 & 19.463 & 0.713 & 1.024 & 6.305 & 5.128 & 0.394 & 11.925 & \\
\hline$P$ value & & $<0.001$ & 0.082 & 0.194 & $<0.001$ & 0.399 & 0.312 & 0.012 & 0.024 & 0.53 & 0.001 & \\
\hline \multicolumn{13}{|l|}{$\begin{array}{l}\text { Subjective } \\
\text { perception of } \\
\text { impacts }\end{array}$} \\
\hline \multicolumn{13}{|l|}{$\begin{array}{l}\text { Epidemic } \\
\text { concern }\end{array}$} \\
\hline None & $411(1.8 \%)$ & $0.10 \pm 0.76$ & $\begin{array}{l}0.13 \pm \\
0.75\end{array}$ & $\begin{array}{l}0.06 \pm \\
0.75\end{array}$ & $\begin{array}{l}-0.14 \pm \\
0.76\end{array}$ & $\begin{array}{l}-0.09 \pm \\
0.76\end{array}$ & $\begin{array}{l}-0.05 \pm \\
0.77\end{array}$ & $\begin{array}{l}-0.03 \pm \\
0.79\end{array}$ & $\begin{array}{l}-0.06 \pm \\
0.76\end{array}$ & $\begin{array}{l}0.17 \pm \\
0.77\end{array}$ & $\begin{array}{l}0.09 \pm \\
2.39\end{array}$ & 1 \\
\hline Low & $3061(13.6 \%)$ & $0.26 \pm 0.76$ & $\begin{array}{l}0.2 \pm \\
0.79\end{array}$ & $\begin{array}{l}0.12 \pm \\
0.80\end{array}$ & $\begin{array}{l}-0.08 \pm \\
0.76\end{array}$ & $\begin{array}{l}0.06 \pm \\
0.84\end{array}$ & $\begin{array}{l}0.01 \pm \\
0.83\end{array}$ & $\begin{array}{l}0.05 \pm \\
0.83\end{array}$ & $\begin{array}{l}0.01 \pm \\
0.82\end{array}$ & $\begin{array}{l}0.19 \pm \\
0.77\end{array}$ & $\begin{array}{l}0.83 \pm \\
3.02\end{array}$ & 2 \\
\hline Medium & $2130(9.5 \%)$ & $0.20 \pm 0.77$ & $\begin{array}{l}0.17 \pm \\
0.78\end{array}$ & $\begin{array}{l}0.08 \pm \\
0.78\end{array}$ & $\begin{array}{l}-0.04 \pm \\
0.72\end{array}$ & $\begin{array}{l}0.12 \pm \\
0.80\end{array}$ & $\begin{array}{l}0.10 \pm \\
0.79\end{array}$ & $\begin{array}{l}0.07 \pm \\
0.80\end{array}$ & $\begin{array}{l}0.03 \pm \\
0.79\end{array}$ & $\begin{array}{l}0.21 \pm \\
0.73\end{array}$ & $\begin{array}{l}0.93 \pm \\
2.88\end{array}$ & 3 \\
\hline High & 7308(32.5\%) & $0.37 \pm 0.67$ & $\begin{array}{l}0.35 \pm \\
0.68\end{array}$ & $\begin{array}{l}0.21 \pm \\
0.70\end{array}$ & $\begin{array}{l}0.20 \pm \\
0.63\end{array}$ & $\begin{array}{l}0.30 \pm \\
0.74\end{array}$ & $\begin{array}{l}0.23 \pm \\
0.74\end{array}$ & $\begin{array}{l}0.26 \pm \\
0.73\end{array}$ & $\begin{array}{l}0.23 \pm \\
0.74\end{array}$ & $\begin{array}{l}0.25 \pm \\
0.62\end{array}$ & $\begin{array}{l}2.40 \pm \\
3.20\end{array}$ & 4 \\
\hline Very high & $9549(42.5 \%)$ & $0.40 \pm 0.66$ & $\begin{array}{l}0.39 \pm \\
0.67\end{array}$ & $\begin{array}{l}0.25 \pm \\
0.69\end{array}$ & $\begin{array}{l}0.26 \pm \\
0.62\end{array}$ & $\begin{array}{l}0.35 \pm \\
0.73\end{array}$ & $\begin{array}{l}0.27 \pm \\
0.74\end{array}$ & $\begin{array}{l}0.31 \pm \\
0.73\end{array}$ & $\begin{array}{l}0.27 \pm \\
0.74\end{array}$ & $\begin{array}{l}0.28 \pm \\
0.61\end{array}$ & $\begin{array}{l}2.8 \pm \\
3.32\end{array}$ & 5 \\
\hline$F$ & & 66.736 & 82.801 & 42.562 & 236.023 & 132.032 & 93.255 & 111.463 & 109.524 & 15.662 & 362.623 & \\
\hline$P$ value & & $<0.001$ & $<.001$ & $\begin{array}{l}<.001 \\
0 .\end{array}$ & $<0.001$ & $<0.001$ & $<0.001$ & $<0.001$ & $<0.001$ & $<0.001$ & $<0.001$ & \\
\hline \multicolumn{13}{|l|}{$\begin{array}{l}\text { Impact of } \\
\text { psychology }\end{array}$} \\
\hline None & 1852(8.2\%) & $0.23 \pm 0.6$ & $\begin{array}{l}0.22 \pm \\
0.61\end{array}$ & $\begin{array}{l}0.15 \pm \\
0.62\end{array}$ & $\begin{array}{l}0.02 \pm \\
0.59\end{array}$ & $\begin{array}{l}0.12 \pm \\
0.7\end{array}$ & $\begin{array}{l}0.07 \pm \\
0.69\end{array}$ & $\begin{array}{l}0.1 \pm \\
0.69\end{array}$ & $\begin{array}{l}0.07 \pm \\
0.69\end{array}$ & $\begin{array}{l}0.13 \pm \\
0.64\end{array}$ & $\begin{array}{l}1.11 \pm \\
2.8\end{array}$ & 1 \\
\hline Low & 7093(31.6\%) & $0.34 \pm 0.67$ & $\begin{array}{l}0.31 \pm \\
0.69\end{array}$ & $\begin{array}{l}0.19 \pm \\
0.7\end{array}$ & $\begin{array}{l}0.1 \pm \\
0.65\end{array}$ & $\begin{array}{l}0.22 \pm \\
0.75\end{array}$ & $\begin{array}{l}0.18 \pm \\
0.74\end{array}$ & $\begin{array}{l}0.2 \pm \\
0.75\end{array}$ & $\begin{array}{l}0.17 \pm \\
0.75\end{array}$ & $\begin{array}{l}0.2 \pm \\
0.65\end{array}$ & $\begin{array}{l}1.91 \pm \\
3.21\end{array}$ & 2 \\
\hline Medium & $7469(33.3 \%)$ & $0.36 \pm 0.7$ & $\begin{array}{l}0.34 \pm \\
0.72\end{array}$ & $\begin{array}{l}0.21 \pm \\
0.73\end{array}$ & $\begin{array}{l}0.17 \pm \\
0.67\end{array}$ & $\begin{array}{l}0.29 \pm \\
0.77\end{array}$ & $\begin{array}{l}0.22 \pm \\
0.76\end{array}$ & $\begin{array}{l}0.25 \pm \\
0.77\end{array}$ & $0.2 \pm 0.77$ & $\begin{array}{l}0.27 \pm \\
0.65\end{array}$ & $\begin{array}{l}2.31 \pm \\
3.27\end{array}$ & 3 \\
\hline High & 4244(18.9\%) & $0.39 \pm 0.73$ & $\begin{array}{l}0.37 \pm \\
0.74\end{array}$ & $\begin{array}{l}0.23 \pm \\
0.77\end{array}$ & $\begin{array}{l}0.26 \pm \\
0.71\end{array}$ & $\begin{array}{l}0.34 \pm \\
0.78\end{array}$ & $\begin{array}{l}0.25 \pm \\
0.8\end{array}$ & $\begin{array}{l}0.3 \pm \\
0.78\end{array}$ & $\begin{array}{l}0.27 \pm \\
0.79\end{array}$ & $\begin{array}{l}0.3 \pm \\
0.67\end{array}$ & $\begin{array}{l}2.7 \pm \\
3.43\end{array}$ & 4 \\
\hline
\end{tabular}




\begin{tabular}{|c|c|c|c|c|c|c|c|c|c|c|c|c|}
\hline Characteristic & $N(\%)$ & Vegetables & $\begin{array}{l}\text { Fresh } \\
\text { Fruits }\end{array}$ & $\begin{array}{l}\text { Meat, } \\
\text { Dairy, } \\
\text { And } \\
\text { Eggs }\end{array}$ & $\begin{array}{l}\text { Salt } \\
\text { Intake }\end{array}$ & $\begin{array}{l}\text { Fried } \\
\text { Foods }\end{array}$ & $\begin{array}{l}\text { Sugary } \\
\text { Foods }\end{array}$ & $\begin{array}{l}\text { Pickled } \\
\text { Fruits }\end{array}$ & $\begin{array}{l}\text { Processed } \\
\text { Meat }\end{array}$ & Drinking & $\begin{array}{l}\text { Total } \\
\text { score }\end{array}$ & $\mathrm{Cc}$ \\
\hline Very high & 1801(8\%) & $0.35 \pm 0.72$ & $\begin{array}{l}0.35 \pm \\
0.73\end{array}$ & $\begin{array}{l}0.22 \pm \\
0.76\end{array}$ & $\begin{array}{l}0.25 \pm \\
0.69\end{array}$ & $\begin{array}{l}0.3 \pm \\
0.79\end{array}$ & $\begin{array}{l}0.21 \pm \\
0.79\end{array}$ & $\begin{array}{l}0.28 \pm \\
0.77\end{array}$ & $\begin{array}{l}0.21 \pm \\
0.79\end{array}$ & $\begin{array}{l}0.31 \pm \\
0.67\end{array}$ & $\begin{array}{l}2.49 \pm \\
3.41\end{array}$ & 5 \\
\hline$F$ & & 19.942 & 15.06 & 4.89 & 69.565 & 35.796 & 19.077 & 28.362 & 25.128 & 36.613 & 96.193 & \\
\hline$P$ value & & $<0.001$ & $<.001$ & 0.001 & $<0.001$ & $<0.001$ & $<0.001$ & $<0.001$ & $<0.001$ & $<0.001$ & $<0.001$ & \\
\hline \multicolumn{13}{|l|}{ Impact of life } \\
\hline None & $1569(7 \%)$ & $0.23 \pm 0.59$ & $\begin{array}{l}0.22 \pm \\
0.61\end{array}$ & $\begin{array}{l}0.13 \pm \\
0.63\end{array}$ & $0 \pm 0.61$ & $\begin{array}{l}0.05 \pm \\
0.69\end{array}$ & $\begin{array}{l}0.04 \pm \\
0.69\end{array}$ & $\begin{array}{l}0.06 \pm \\
0.69\end{array}$ & $\begin{array}{l}0.02 \pm \\
0.68\end{array}$ & $\begin{array}{l}0.12 \pm \\
0.65\end{array}$ & $\begin{array}{l}0.87 \pm \\
2.73\end{array}$ & 1 \\
\hline Low & $5995(26.7 \%)$ & $0.33 \pm 0.69$ & $\begin{array}{l}0.30 \pm \\
0.70\end{array}$ & $\begin{array}{l}0.19 \pm \\
0.71\end{array}$ & $\begin{array}{l}0.08 \pm \\
0.68\end{array}$ & $\begin{array}{l}0.20 \pm \\
0.78\end{array}$ & $\begin{array}{l}0.16 \pm \\
0.76\end{array}$ & $\begin{array}{l}0.18 \pm \\
0.78\end{array}$ & $\begin{array}{l}0.15 \pm \\
0.77\end{array}$ & $\begin{array}{l}0.22 \pm \\
0.68\end{array}$ & $\begin{array}{l}1.81 \pm \\
3.24\end{array}$ & 2 \\
\hline Medium & $7780(34.6 \%)$ & $0.35 \pm 0.70$ & $\begin{array}{l}0.33 \pm \\
0.72\end{array}$ & $\begin{array}{l}0.19 \pm \\
0.73\end{array}$ & $\begin{array}{l}0.17 \pm \\
0.67\end{array}$ & $\begin{array}{l}0.28 \pm \\
0.77\end{array}$ & $\begin{array}{l}0.22 \pm \\
0.76\end{array}$ & $\begin{array}{l}0.25 \pm \\
0.76\end{array}$ & $\begin{array}{l}0.21 \pm \\
0.77\end{array}$ & $\begin{array}{l}0.25 \pm \\
0.65\end{array}$ & $\begin{array}{l}2.24 \pm \\
3.27\end{array}$ & 3 \\
\hline High & $5046(22.5 \%)$ & $0.40 \pm 0.70$ & $\begin{array}{l}0.37 \pm \\
0.72\end{array}$ & $\begin{array}{l}0.24 \pm \\
0.74\end{array}$ & $\begin{array}{l}0.25 \pm \\
0.66\end{array}$ & $\begin{array}{l}0.36 \pm \\
0.75\end{array}$ & $\begin{array}{l}0.26 \pm \\
0.77\end{array}$ & $\begin{array}{l}0.30 \pm \\
0.75\end{array}$ & $\begin{array}{l}0.26 \pm \\
0.76\end{array}$ & $\begin{array}{l}0.31 \pm \\
0.63\end{array}$ & $\begin{array}{l}2.77 \pm \\
3.34\end{array}$ & 4 \\
\hline Very high & $2069(9.2 \%)$ & $0.36 \pm 0.7$ & $\begin{array}{l}0.35 \pm \\
0.71\end{array}$ & $\begin{array}{l}0.21 \pm \\
0.73\end{array}$ & $\begin{array}{l}0.26 \pm \\
0.64\end{array}$ & $\begin{array}{l}0.33 \pm \\
0.75\end{array}$ & $\begin{array}{l}0.22 \pm \\
0.77\end{array}$ & $\begin{array}{l}0.28 \pm \\
0.75\end{array}$ & $\begin{array}{l}0.21 \pm \\
0.77\end{array}$ & $\begin{array}{l}0.29 \pm \\
0.63\end{array}$ & $\begin{array}{l}2.51 \pm \\
3.31\end{array}$ & 5 \\
\hline$F$ & & 18.433 & 16.8 & 8.869 & 82.693 & 66.943 & 33.35 & 41.176 & 36.138 & 31.173 & 130.765 & \\
\hline$P$ value & & $<0.001$ & $<0.001$ & $<.001$ & $<0.001$ & $<0.001$ & $<0.001$ & $<0.001$ & $<0.001$ & $<0.001$ & $<0.001$ & \\
\hline \multicolumn{13}{|l|}{$\begin{array}{l}\text { Impact of } \\
\text { work or study }\end{array}$} \\
\hline None & $1702(7.6 \%)$ & $0.22 \pm 0.60$ & $\begin{array}{l}0.21 \pm \\
0.62\end{array}$ & $\begin{array}{l}0.13 \pm \\
0.63\end{array}$ & $\begin{array}{l}-0.02 \pm \\
0.60\end{array}$ & $\begin{array}{l}0.05 \pm \\
0.70\end{array}$ & $\begin{array}{l}0.04 \pm \\
0.69\end{array}$ & $\begin{array}{l}0.08 \pm \\
0.70\end{array}$ & $\begin{array}{l}0.01 \pm \\
0.69\end{array}$ & $\begin{array}{l}0.13 \pm \\
0.66\end{array}$ & $\begin{array}{l}0.85 \pm \\
2.77\end{array}$ & 1 \\
\hline Low & $5767(25.7 \%)$ & $0.32 \pm 0.70$ & $\begin{array}{l}0.29 \pm \\
0.72\end{array}$ & $\begin{array}{l}0.19 \pm \\
0.73\end{array}$ & $\begin{array}{l}0.07 \pm \\
0.70\end{array}$ & $\begin{array}{l}0.18 \pm \\
0.79\end{array}$ & $\begin{array}{l}0.15 \pm \\
0.78\end{array}$ & $\begin{array}{l}0.16 \pm \\
0.79\end{array}$ & $\begin{array}{l}0.13 \pm \\
0.78\end{array}$ & $\begin{array}{l}0.21 \pm \\
0.69\end{array}$ & $\begin{array}{l}1.71 \pm \\
3.26\end{array}$ & 2 \\
\hline Medium & 7537(33.6\%) & $0.35 \pm 0.71$ & $\begin{array}{l}0.33 \pm \\
0.72\end{array}$ & $\begin{array}{l}0.19 \pm \\
0.73\end{array}$ & $\begin{array}{l}0.17 \pm \\
0.68\end{array}$ & $\begin{array}{l}0.27 \pm \\
0.77\end{array}$ & $\begin{array}{l}0.21 \pm \\
0.76\end{array}$ & $\begin{array}{l}0.25 \pm \\
0.77\end{array}$ & $\begin{array}{l}0.22 \pm \\
0.76\end{array}$ & $\begin{array}{l}0.26 \pm \\
0.65\end{array}$ & $\begin{array}{l}2.24 \pm \\
3.25\end{array}$ & 3 \\
\hline High & $4948(22 \%)$ & $0.41 \pm 0.69$ & $\begin{array}{l}0.39 \pm \\
0.71\end{array}$ & $\begin{array}{l}0.24 \pm \\
0.73\end{array}$ & $\begin{array}{l}0.26 \pm \\
0.65\end{array}$ & $\begin{array}{l}0.38 \pm \\
0.74\end{array}$ & $\begin{array}{l}0.28 \pm \\
0.76\end{array}$ & $\begin{array}{l}0.31 \pm \\
0.75\end{array}$ & $\begin{array}{l}0.27 \pm \\
0.76\end{array}$ & $\begin{array}{l}0.29 \pm \\
0.63\end{array}$ & $\begin{array}{l}2.84 \pm \\
3.35\end{array}$ & 4 \\
\hline Very high & $2505(11.2 \%)$ & $0.37 \pm 0.68$ & $\begin{array}{l}0.36 \pm \\
0.68\end{array}$ & $\begin{array}{l}0.22 \pm \\
0.71\end{array}$ & $\begin{array}{l}0.27 \pm \\
0.61\end{array}$ & $\begin{array}{l}0.35 \pm \\
0.74\end{array}$ & $\begin{array}{l}0.24 \pm \\
0.74\end{array}$ & $\begin{array}{l}0.29 \pm \\
0.72\end{array}$ & $\begin{array}{l}0.23 \pm \\
0.75\end{array}$ & $\begin{array}{l}0.29 \pm \\
0.61\end{array}$ & $\begin{array}{l}2.62 \pm \\
3.22\end{array}$ & 5 \\
\hline$F$ & & 26.263 & 27.229 & 9.863 & 103.28 & 89.873 & 40.707 & 48.378 & 49.957 & 27.537 & 166.011 & \\
\hline P value & & $<0.001$ & $\begin{array}{l}<.001 \\
0.01\end{array}$ & $\begin{array}{l}< \\
0.001\end{array}$ & $<0.001$ & $<0.001$ & $<0.001$ & $<0.001$ & $<0.001$ & $<0.001$ & $<0.001$ & \\
\hline Overall & $22459(100 \%)$ & $0.35 \pm 0.69$ & $\begin{array}{l}0.33 \pm \\
0.71\end{array}$ & $\begin{array}{l}0.2 \pm \\
0.72\end{array}$ & $\begin{array}{l}0.16 \pm \\
0.67\end{array}$ & $\begin{array}{l}0.26 \pm \\
0.77\end{array}$ & $\begin{array}{l}0.2 \pm \\
0.76\end{array}$ & $\begin{array}{l}0.23 \pm \\
0.76\end{array}$ & $\begin{array}{l}0.19 \pm \\
0.76\end{array}$ & $\begin{array}{l}0.25 \pm \\
0.66\end{array}$ & $\begin{array}{l}2.17 \pm \\
3.28\end{array}$ & \\
\hline
\end{tabular}

Code: code in stepwise linear regression models

\subsection{Baseline of dietary habits, and willingness to change dietary habits}

The mean score of intakes of vegetables, fresh fruits was $-1.53,-1.88$, respectively, while the mean score of intakes of salt, fried foods, sugary foods, pickled fruits, processed meat were more than 2. Otherwise, the mean score of DBI_HBS, DBI_LBS and DBI_TS and was18.01, -5.44 and 12.56, respectively (Fig. 1). The difference in baseline dietary habits between different demographic characteristics was statistically significant (all $P$ values $<0.05$ ).

The mean score of change willingness of each food group was about 0.20 , and the mean level of total score of willingness to change dietary habits was 0.22 (ranges from -9 to 9). The difference in willingness to change dietary habits between different demographic characteristics was statistically significant (all $P$ values $<0.05$ ) (Table 2, Fig. 2).

\subsection{Subjective perception of impact and willingness to change dietary habits}

For both of univariate and multivariable analysis of associations between subjective perception of impact and willingness to change dietary habits, those who self-reported higher epidemic concerns and higher impacts on psychology and work were more likely to have higher score willingness to change dietary habits $(\mathrm{P}<0.05)$. Furthermore, female, the older, normal BMI, non-medical worker, higher education level, and married were also significantly associated with a higher score of willingness to adopt healthy dietary habits. $(P<0.05)$ (Table 2, Table 3$)$. 
Table 3

Associations between subjective perception of impact and willingness to change dietary habits

\begin{tabular}{|c|c|c|}
\hline Characteristics & b & $P$ value \\
\hline \multicolumn{3}{|l|}{ General characteristics } \\
\hline \multicolumn{3}{|l|}{ Gender } \\
\hline Male(Ref) & - & - \\
\hline Female & 0.507 & $<0.001$ \\
\hline Age(Year) & 0.292 & $<0.001$ \\
\hline \multicolumn{3}{|l|}{ BMI } \\
\hline $18.5-24$ (Ref) & - & - \\
\hline$<18.5$ & -0.056 & $<0.001$ \\
\hline$\geq 24$ & -0.265 & 0.247 \\
\hline Mainly Living Place & - & - \\
\hline Education Level & 0.183 & $<0.001$ \\
\hline \multicolumn{3}{|l|}{ Arital Status } \\
\hline Married (Ref) & - & - \\
\hline Unmarried & -0.344 & $<0.001$ \\
\hline \multicolumn{3}{|l|}{ Occupation } \\
\hline Medical Workers (Ref) & - & - \\
\hline Others & 0.455 & $<0.001$ \\
\hline \multicolumn{3}{|c|}{ Subjective perception of impacts } \\
\hline Epidemic concern & 1.144 & $<0.001$ \\
\hline Impact of psychology & 0.195 & $<0.001$ \\
\hline Impact of life & 0.011 & 0.199 \\
\hline Impact of work or study & 0.411 & $<0.001$ \\
\hline DBI_TS & 0.068 & $<0.001$ \\
\hline DBI_LBS & -0.024 & 0.047 \\
\hline DBI_HBS & -0.186 & $<0.001$ \\
\hline
\end{tabular}

\section{Discussion}

In this study, we examined the associations between impact of the COVID-19 pandemic and willingness to adopt healthy dietary habits among Chinese local residents. We found that mean score of willingness to change dietary habits was 2.2 ( ranges from - 9 to 9 ), indicating a positive improvement to proper diet. Higher epidemic concern, and higher impact on psychology, work, as well as female, older, normal BMI, higher education level, married were significantly associated with higher willingness score. Our findings added to the evidence on the characteristic of change in diet during the pandemic of COVID-19. Our results revealed that Chinese local residents with multiple higher perception of epidemic concern, or impact on psychology, work have a much greater willingness to adopt healthy dietary habits and need special attention and support from management department and healthcare providers. This is important because individual level behavior change is guided by both subjective and objective risks and because perceptions of impact may act as a conditioning factor in a participant's balancing of concerns of safety, self-protection and health during the pandemic ${ }^{12}$.

Chen Yan et al. reported that compared to lifestyles of " no gathering and less going out ", "wearing masks when going out" and "not going to crowded and closed places" etc., the ratio of residents that could achieve the lifestyle of "light diets with balanced portion of vegetables and meat" was relatively low (65.6\%) after outbreak of COVID-19 ${ }^{13}$. Food consumption and meal patterns (the type of food, eating out of control, snacks between meals, number of meals) were more unhealthy during confinement due to COVID-19 pandemic ${ }^{14}$. The negative changes in the majority of eating behaviors could be attributed to eating out of anxiety or boredom ${ }^{15}$, a dip in motivation to participate in physical activity or maintain healthy eating ${ }^{16}$ or an increase in anxiety or mood driven eating ${ }^{15}$. However, we found that people showed a generally positive willingness to adopt habits of healthy diets, implying the improvement for dietary behavior was mainly at the former stages (precontemplation, contemplation, preparation). Thus, it is necessary to take specific measures and interventions for the current stages of behavior to promote to a higher stage. 
It is not clear how Chinese adults would change their diet after the epidemic is gradually under control, but the impact of the pandemic persisting for a protracted period could lead to prolonged influence of lifestyle including dietary habits in a substantial proportion of the population. It is well established that unhealthy dietary habits are associated with non-communicable chronic diseases such as hypertension, diabetes and obesity and with premature mortality ${ }^{5}$. Both of morbidity ${ }^{3}$ and mortality ${ }^{4}$ of COVID-19 among those with underlying medical conditions are much higher than healthy individuals. Furthermore, dietary habits may play an important role in the long-term recovery of COVID-19 cases ${ }^{7}$. Targeting diet health messaging to address the potential harms of subjective risks may also be key, given that those who have no known objective clinical risk in the current epidemic may change diet behavior in light of their perception of concern or impact, thereby driving the development of clinical risk factors and as a consequence potentially suffering more severe sequelae of COVID-19 infections during future epidemic ${ }^{12}$. The results of our current study suggest that the general public who have higher perception of epidemic concern, or of impact on psychology, work may be disproportionately impacted on dietary habits because they are more likely to reduce the unhealthy habits of their diet. Scientists have published recommendations of eating well during COVID-19 for different groups including the general population, the elderly, children, pregnant women and chronic patients, which improve their health status in case they contract coronavirus and become severely ill. This advice may be even more pertinent for those who are at lower perception of concern or impact by COVID-19 on psychology and work. Our findings suggest that these subpopulations are less likely to adopt healthy dietary habits than before the epidemic. To counteract poor dietary behaviors, meal planning and opting for healthy alternatives is the best approach to combating unhealthy eating habits while in confinement ${ }^{15}$. Alternative support for motivation during the pandemic may be sourced from multi-sector's cooperation including health system and assistive technologies such as apps, streaming services and social media. New advices that promote simple and feasible diet plans should be considered as part of any new public health guidelines for future prevention and control measures.

Female, the older, normal BMI, non-medical worker, higher education level, and married were also significantly associated with a higher score of willingness to adopt healthy dietary habits. More female than male respondents were likely to promote diet modification, which was consistent with several studies conducted among different age groups ${ }^{17}$. This may be because in China, there is a tradition that dietary cooking is generally undertaken by women. Therefore, female residents pay more attention to diet and have more knowledge of healthy diet than male residents ${ }^{18}$. With the growth of age, health literacy is improving and become more rich and comprehensive ${ }^{19}$. Notably, for youth about 20-30 ages, ideas and behaviors can be adopted and formed firmly as they are more receptive to new knowledge including diet-related education ${ }^{20}$. Not surprisingly, respondents who were at a higher grade had a good academic performance had higher knowledge and belief about healthy diets compared with the others ${ }^{19}$. Furthermore, those married significantly increased the score of willingness, which suggested that dining with family has a positive effect on improving their eating habits ${ }^{21}$. Surprisingly, medical staffs were associated with a lower willingness score in our sample, suggesting that increased attention should be also paid to the knowledge and attitudes of medical staff. Additionally, people with normal BMI had higher level of willingness score, but no significant difference was found between individuals with high BMI and normal BMI.

Recent researches had showed that obesity is a risk factor of covid-19, and the proportion of severe disease is higher than those with normal $\mathrm{BMI}{ }^{22}$. Therefore, corresponding health intervention measures should be carried out for sub-populations of male, lower age, abnormal BMI especially obesity, lower education level, unmarried and medical staff.

To date, studies examining changes in dietary habits before and during COVID-19 pandemic are limited in number. This is the first study using data in China to evaluate willingness to adopt healthy dietary habits during the COVID-19 pandemic. Further research should address (1) insight into sub-populations, for the development of bespoke interventions to address their needs, and (2) interference of diet base on characteristics of the behavior among different subpopulations, for improving interventions. However, our study had several notable limitations. Firstly, the findings from this brief cross-sectional study are only suggestive (not confirmative) for causal associations between subjective impact and willingness change to dietary habits. Secondly, all indicators were based on an online survey of respondents' self-reports and were thus subject to recall and report bias. However, according to baseline of dietary habits, the intakes of vegetables, fresh fruits, dairy etc. were still not enough, while salt, fried foods, sugary foods intake etc. were high disproportionately, which was in line with the imbalanced dietary pattern of China Health and Nutrition Survey (CHNS) ${ }^{23}$. Thirdly, we did not use standardized scales to assess subjective perception of impact (e.g., epidemic concern and impacts on psychology, life, work, or study during the COVID-19 outbreak) and baseline and change willingness of dietary habits (e.g., the status of salt intake, the frequency of eating fried foods, and sugary foods) due to a limited survey time. Lastly, we might have missed some groups lacking interest in these kinds of online surveys or lacking access to a social media. Although this convenience sample is not representative of the public at large, the anonymity, confidentiality, and sample size of the data may partially overcome the factors mentioned above.

\section{Conclusions}

In conclusion, the results of this study indicated a generally positive improvement to a proper diet among Chinese people. The impact of the COVID-19 pandemic was associated with a higher score of willingness to adopt healthy dietary habits among female, the older, non-medical worker, and individuals married or with higher education level, normal BMI. Taking into account the changing features of diets may help nutritional recommendations to maintain health, and prevent and control COVID-19 during the pandemic period.

\section{Declarations}

\section{Ethics approval and consent to participate}

This study was approved by the ethics committee of Guangdong Medical University. Because we all use online questionnaires, the consent obtained from participants is only an option before the beginning of the questionnaire. If the participants do not agree, the questionnaire will not be viewable.Data used in this study is a public open resource and does not need any administrative permissions.

Consent for publication

Page 10/13 
Not applicable.

\section{Competing interests}

The authors declare that they have no competing interests.

\section{Funding}

Funds for PHD researchers of Guangdong Medical University in 2020(GDMUB2020013).

COVID-19 pandemic prevention and control special research project for University of Guangdong Province in 2020(2020KZDZX1099).The funding body had no role in the design of the study, the collection, analysis, and interpretation of data or the writing of the manuscript.

\section{Authors' contributions}

Ying Xu and Zhi-xue Li designed the whole process and were the main drafters of the manuscript. Weijun Yu, Ting Liu, Zheng Liu, Liuna Yang, Ailun xie, Zhenzhu Qian and Rencheng Zhao did some of the expeditionary research and contributed the coordination and manuscript editing. Yan-Fang Guo and Jialong Chen were the directors for the fund and designed ideas of research. All participated in the analysis and discussion under the leadership and instruction of Jia-long Chen. All authors read and approved the final manuscript.

\section{Availability of data and material}

All data in the study can be accessed from the corresponding author up on request.

\section{Ethics approval and consent to participate}

Ethical approval for the study was obtained from the Guangdong Medical University Ethics Committees.

\section{References}

1. Coronavirus disease. 2019 (COVID-19) Situation Report-87: World Health Organization, 2020.

2. WHO Director-General's opening remarks at the media briefing on COVID-19-11. March 2020. 2020. https://www.who.int/zh/dg/speeches/detail/whodirector-general-s-opening-remarks-at-the-media-briefing-on-covid-19--11-march-2020 (accessed 11 March 2020).

3. Zhang Y. The epidemiology characteristics of an outbreak of 2019 novel coronavirus disease (COVID-19). Chinese Journal of Preventive Medicine. 2020;02:145-51.

4. Guan W, Liang W, Zhao Y, et al. Comorbidity and its impact on 1,590 patients with COVID-19 in China: A nationwide analysis. MedRxiv 2020.

5. Colpani V, Baena CP, Jaspers L, et al. Lifestyle factors, cardiovascular disease and all-cause mortality in middle-aged and elderly women: a systematic review and meta-analysis. Eur J Epidemiol. 2018;33(9):831-45.

6. Hamer M, Kivimäki M, Gale CR, Batty GD. Lifestyle risk factors for cardiovascular disease in relation to COVID-19 hospitalization: A community-based cohort study of 387,109 adults in UK. MedRxiv 2020.

7. Butler MJ, Barrientos RM. The impact of nutrition on COVID-19 susceptibility and long-term consequences. Brain, behavior, and immunity 2020.

8. Nelson LM, Simard JF, Oluyomi A, et al. US public concerns about the COVID-19 pandemic from results of a survey given via social media. JAMA internal medicine 2020.

9. Kwok KO, Li KK, Chan HH, et al. Community responses during the early phase of the COVID-19 epidemic in Hong Kong: Risk perception, information exposure and preventive measures. MedRxiv 2020.

10. Willett WC, Koplan JP, Nugent R, Dusenbury C, Gaziano TA. Prevention of chronic disease by means of diet and. lifestyle changes: PubMed; 2006.

11. Petrocelli JV. Processes and stages of change: Counseling with the Transtheoretical Model of change. Journal of Counseling \& Development 2002; 80(1).

12. Rogers NT, Waterlow N, Brindle H, et al. Behavioural change towards reduced intensity physical activity is disproportionately prevalent among adults with serious health issues or self-perception of high risk during the UK COVID-19 lockdown. MedRxiv 2020.

13. Chen Y, Jin Y, Zhu L, et al. The network investigation on knowledge, attitude and practice about COVID-19 of the residents in Anhui province. Chinese Journal of Preventive Medicine 2020; (04): E004-E.

14. Ammar A, Brach M, Trabelsi K, Chtourou H. Effects of COVID-19 home confinement on physical activity and eating behaviour Preliminary results of the ECLB-COVID19 international online-survey. MedRxiv 2020.

15. BDA. Eating well during Coronavirus / COVID-19. 2020. https://www.bda.uk.com/resource/eating-well-during-coronavirus-covid-19.html (accessed April $16,2020$.

16. AL BG R. Habit formation and behavior change. 2019. https://uni-muenster.sciebo.de/apps/files/? dir=/2020_PROCare4Life_3100050300/02_Online\%20survey\%20ECLB-COVID19\&fileid=1748656124\#pdfviewer (accessed May 16, 2020).

17. He Y, Li Y, Lai J, et al. Dietary patterns as compared with physical activity in relation to metabolic syndrome among Chinese adults. Nutrition Metabolism Cardiovascular Diseases. 2013;23(10):920-8.

18. Yan W, Liping M, Haotian F, Ting L. Nutrition knowledge of elderly and middle-age urban population and its effects on diet quality and dairy consumption: A Cross-sectional study in eight cities of China (P10-060-19). Current Developments in Nutrition 2019; (Supplement_1): Supplement_1. 
19. Cai ZY, Chen T, Wei L, Ping G, Jian X, Chen BD. Survey and Analysis on Knowledge, Attitude and Practice Status Quo of Balanced Nutrition among Residents in Baoshan District. Health Education \& Health Promotion 2007.

20. Al-Mahrooqi B, Al-Hadhrami R, Al-Amri A, Al-Tamimi S, Al-Ghafri T. Self reported knowledge of diabetes among high school students in Al-Amerat and Quriyat, Muscat Governate, Oman. Sultan Qaboos University Medical Journal. 2013;13(3):392-8.

21. Wang LL, Lin D, Shao YQ, et al. An investigation on knowledge,attitude,behavior about nutrition among elderly residents in 2 communities in Wenzhou City. Preventive Medicine 2017.

22. Simonnet A, Chetboun M, Poissy J, et al. High prevalence of obesity in severe acute respiratory syndrome coronavirus-2 (SARS-CoV-2) requiring invasive mechanical ventilation. Obesity (Silver Spring, Md) 2020.

23. Yu-na H, Feng-ying Z, Ke-you G. Measuring diet quality of chinese 18-59 years adult using chinese diet balance index. Journal of Hygiene Research. 2005;4(34):442.

\section{Figures}

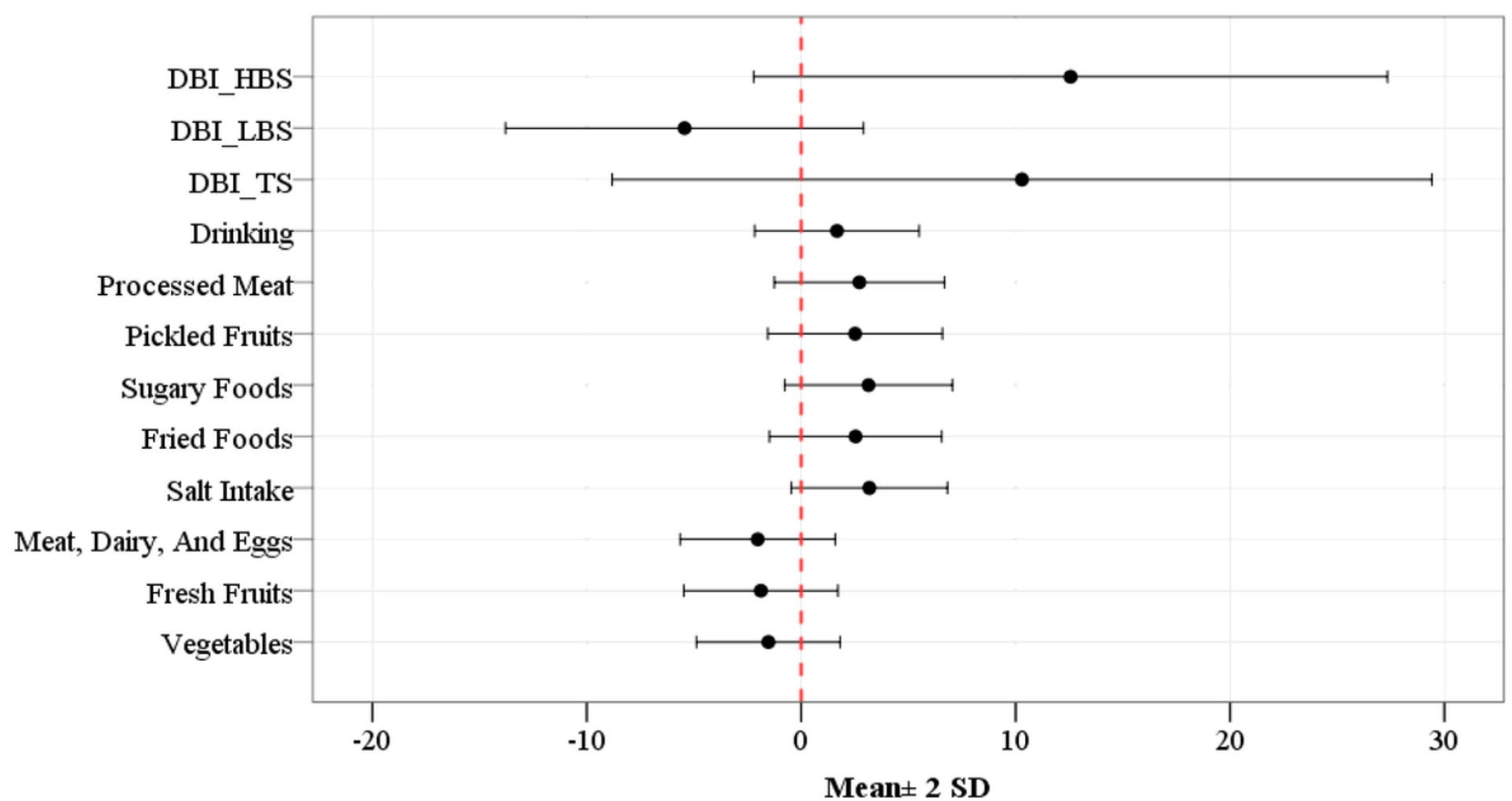

Figure 1

Mean score of dietary habits before the outbreak of COVID-19 


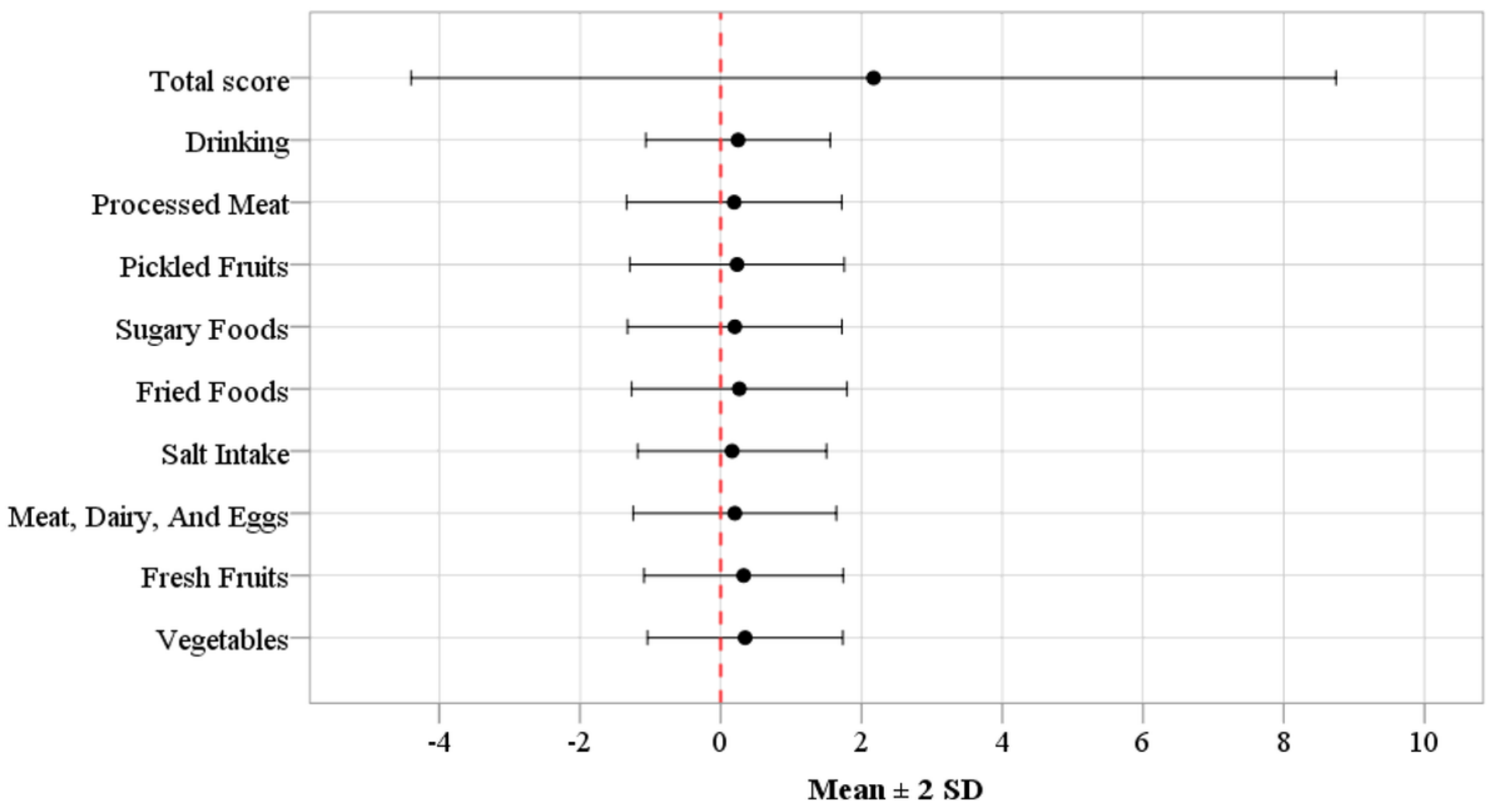

Figure 2

Willingness to change dietary habits after the outbreak of COVID-19

\section{Supplementary Files}

This is a list of supplementary files associated with this preprint. Click to download.

- renamed98860.doc 